\title{
Brugada Pattern: Unraveling Possible Cardiac Manifestation of SARS-CoV-2 Infection
}

\author{
Maria Teresa Boncoraglio ${ }^{\mathrm{a}, \mathrm{c}}$, Joana Esteves ${ }^{\mathrm{a}}$, Francisca Pereira ${ }^{\mathrm{a}}$, Joana Braga ${ }^{\mathrm{a}}$, \\ Carolina Veiga ${ }^{\mathrm{a}}$, Daniel G. Oliveira ${ }^{\mathrm{b}}$, Pilar Barbeito ${ }^{\mathrm{a}}$
}

\begin{abstract}
We report the case of a 41-year-old patient with no family history of sudden cardiac death. The patient presented with high fever and vomiting and was diagnosed with acute pyelonephritis. Screening for severe acute respiratory syndrome coronavirus 2 (SARS-CoV-2) infection was positive. An electrocardiogram (ECG) performed during a fever episode revealed a Brugada pattern. Fever can be a trigger for induction of the electrocardiographic Brugada pattern but it is still unknown if the cardiac involvement by coronavirus disease 2019 (COVID-19) can interfere with myocardial ion channels.
\end{abstract}

Keywords: Brugada pattern; COVID-19; Fever; Pyelonephritis

\section{Introduction}

The Brugada syndrome is an autosomal dominant genetic disorder characterized by a specific electrocardiogram (ECG) pattern associated with an increased risk of ventricular arrhythmias and sudden cardiac death in a structurally normal heart [1].

The hallmark of Brugada syndrome is characteristic electrocardiographic pattern, which can often be dynamic and sometimes concealed, induced by certain situations, such as fever, vagal stimulation, and electrolyte disturbance. Furthermore, the concept of Brugada phenocopy (or acquired form) exists in which Brugada pattern may appear in relation with multiple causes (metabolic conditions, ischemia, myocardial/ pericardial diseases, etc.), and disappear after resolution of the injury that caused it, in the absence of true congenital Brugada syndrome $[2,3]$.

Coronavirus disease 2019 (COVID-19) is associated with

Manuscript submitted January 9, 2021, accepted January 21, 2021

Published online March 5, 2021

aHospital Santa Maria Maior, Barcelos, Portugal

${ }^{b}$ Centro Hospitalar e Universitario do Porto, Porto, Portugal

${ }^{\mathrm{c}}$ Corresponding Author: Maria Teresa Boncoraglio, Hospital Santa Maria

Maior, Barcelos, Portugal. Email: mariateresab87@gmail.com

doi: https://doi.org/10.14740/jmc3644 a high incidence of cardiac arrhythmias possibly caused by a direct insult of the virus to cardiac myocytes and systemic illness [4].

We report a case of a man with acute pyelonephritis and COVID-19 in which an ECG performed during a feverish peak revealed a coved ST-segment elevation in lead V1 in a patient with previously unrecognized Brugada syndrome.

\section{Case Report}

A 41-year-old male presented to our Emergency Department with a 3-day history of unremitting fever, nausea, vomit, myalgia and sneezing. He had a history of autism and his medications included benzodiazepines. There was no history of familiar sudden death or arrhythmic diseases.

His vital signs showed a blood pressure of 129/69 mm Hg, heart rate of 98 beats $/ \mathrm{min}$, respiratory rate of 24 breaths/min, $\mathrm{O}_{2}$ saturation of $97 \%$ on room air, and a temperature of 39.4 ${ }^{\circ} \mathrm{C}$.

On examination, the patient had equal breath sounds bilaterally without wheezing, stridor, rales, or rhonchi; heart rate was regular with normal S1 and S2 without murmurs, rubs, or gallops; the abdomen was depressible. A doubtful renal Murphy's punch sign was present.

Laboratory data demonstrated an elevated C-reactive protein level (119 mg/L), leukocytosis with neutrophilia, without leukopenia. High-sensitivity cardiac troponin $\mathrm{T}$ and brain natriuretic peptide were within the normal range. His electrolytes were normal. Urinary sediment had leukocyturia and was positive for nitrites.

The thoracic computed tomography (CT) was normal and the abdominal CT showed pyelic distention without identifiable lithiasis.

A standard nasopharyngeal swab was performed and resulted positive for severe acute respiratory syndrome coronavirus 2 (SARS-CoV-2). Because of the persisting vomiting and the fact that this autistic patient had difficulty expressing himself an ECG was performed, while febrile, revealing a sinus rhythm and coved ST-segment elevation in lead V1 (type 1 Brugada pattern) (Fig. 1).

The patient was admitted for acute pyelonephritis, type 1 Brugada pattern and COVID-19.

He was placed in a dedicated COVID-19 unit with telemetry monitoring. He started antibiotic treatment with ceftri- 


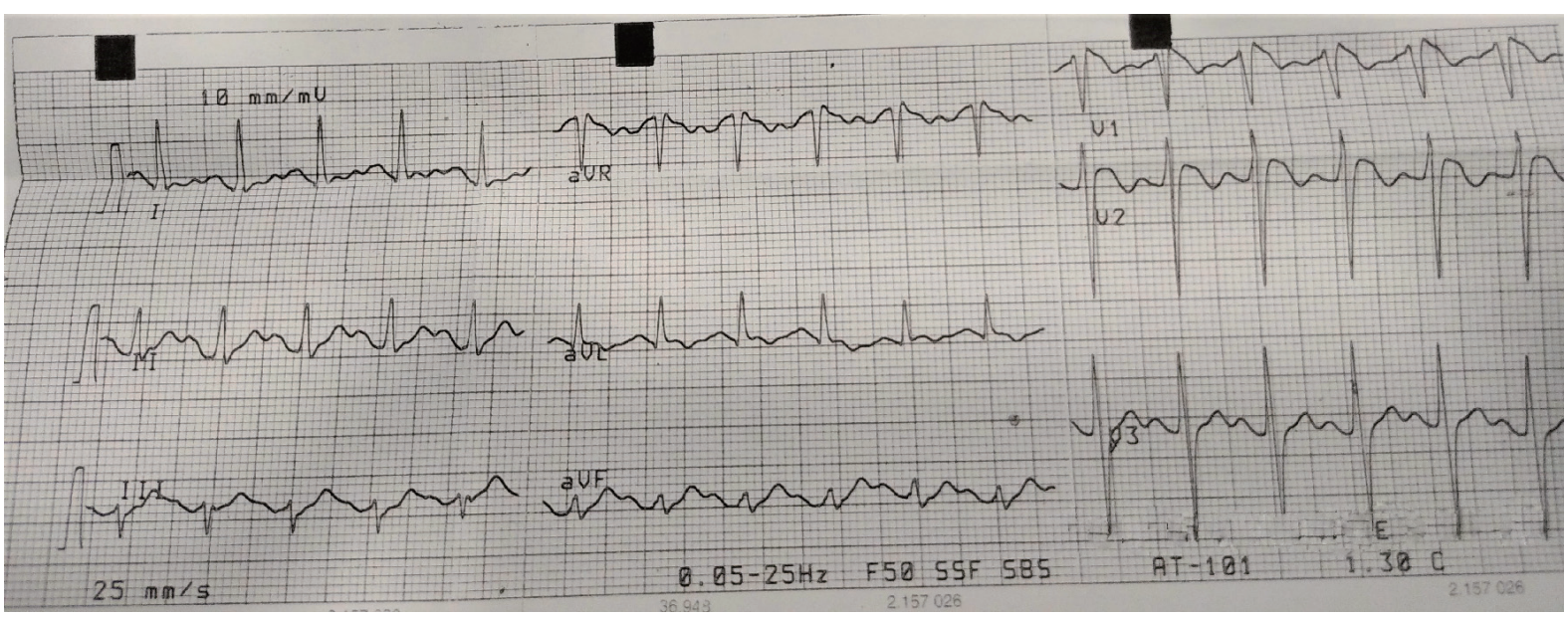

Figure 1. Electrocardiogram in the emergency department with fever of $39.4{ }^{\circ} \mathrm{C}$, shows coved ST elevation in leads V1 and V2 consistent in type 1 Brugada pattern.

axone. Fever was promptly treated with antipyretic therapy. Drugs contraindicated in Brugada syndrome were avoided. The patient did not require supplemental oxygen to maintain arterial saturation.

During monitoring there were no records of either syncope or arrhythmias. The patient was monitored in the telemetry ward until the fever is resolved. All serial troponin values were negative.

Echocardiography showed no structural heart disease.

The ECG performed before discharge showed sinus rhythm 70 beats per minute with complete resolution of the initial, transient Brugada ECG pattern.

\section{Discussion}

The Brugada pattern is one of several electrocardiographic patterns characterized by incomplete right bundle-branch block and ST elevations in the anterior precordial leads. If the Brugada pattern is associated with clinical findings (nonvagal syncope, seizure, nocturnal agonal respiration, polymorphic ventricular tachycardia or sudden death) or familial antecedents of sudden death, patients are said to have Brugada syndrome [1]. In addition, electrocardiographic patterns identical to Brugada pattern are observed in patients with acquired forms, induced by many circumstances including myocardial and pericardial diseases [3].

COVID-19 is a newly recognized infectious disease presenting mainly with fever, pneumonia, and respiratory failure. It is associated with an increased risk of myocardial injury, myocarditis, acute coronary syndromes, and dysrhythmia $[4$, 5]. In particular, a considerable portion of patients who presented with ST-segment elevation myocardial infarction have no obvious thrombus or coronary flow interruption [6].

Evidence suggests that hospitalized patients with COVID-19 experience arrhythmic complications, especially those admitted to intensive care unit [7]. The underlying mechanism that increase the risk of arrhythmias, among others, may be mediated by hypoxia result of lung injury, myocarditis, an ab- normal host immune response through the production of inflammatory cytokines, myocardial ischemia and strain [8].

Fever is a very well-known trigger of Brugada pattern and can precipitate arrhythmia in patients with Brugada syndrome. Temperature increases induce some degree of inactivation of both mutated and wild sodium channels leading to reduced sodium flow responsible for ventricular arrhythmic events [9].

As of this moment, several case reports were published that describe patients with Brugada pattern in the setting of COVID-19.

In the two cases of COVID-19 reported by van de Poll, both patients developed a type 1 Brugada electrocardiographic pattern during fever and did not experience any type of arrhythmia although one of the two had a previous syncope episode [10].

Mahadevaiah and colleagues have also described a 40-year-old patient with fever of $39.1^{\circ} \mathrm{C}$ and chest pain, his ECG showing a type 1 Brugada pattern with normal troponin level and electrolytes [11] like our case.

On the other hand, an Italian author reported a case of 57-year-old man admitted with COVID-19, dyspnoea and chest pain with an ECG revealing type 1 Brugada pattern with no clear relation with fever [12].

Lugenbiel et al described a 53-year-old female with COVID-19, whose ECG with fever shows type 1 Brugada pattern, worsening (progressive increased J-point elevation) as the disease progresses regardless of body temperature. ECG changes resolved with the improvement of the disease. Of note, a previous ECG was normal, as were troponin and electrolytes [13].

None of the patients described had family history of sudden death or arrhythmic diseases. Although Brugada syndrome has a variable penetrance, it is autosomal dominant in transmission [1]. We do not know how first-degree relatives were screened in those studies, but if the Brugada pattern in the reported patients were due to a genetic mutation we would expect at least some cases of familial association. On the other hand, the low number of patients reported with Brugada pattern and COVID-19 makes it difficult to extrapolate. 
The existing evidence, although small, raises the question of whether or not the cases of Brugada patterns in the absence of clear triggers, including fever, could also be caused by COVID-19 infection. Admitting that any disease causing fever can unmask a Brugada pattern, it cannot be excluded that a direct cardiotoxicity of the virus may be responsible for typical changes in the ECG.

We report on a single patient and extrapolate from indirect and non-invasive testing. The other few cases reported in the literature, however, consubstantiate this potential association of COVID-19 and electrocardiographic changes. Additionally, the characteristic elusive detection of Brugada pattern raises the question of whether this condition is not much more prevalent in COVID-19 patients. Further studies must be undertaken to more clearly address this issue.

\section{Conclusions}

This case describes the development of Brugada pattern in the setting of an acute febrile illness due to pyelonephritis and COVID-19.

It is still unknown if SARS-CoV-2 has a direct effect on myocardiocytes. In addition to our case only a handful of other provide evidence for this potential association, but if proven, could have implications for many patients. We believe that it remains important for physicians treating this novel infection by SARS-CoV-2 to remain vigilant and with a high degree of suspicion for more uncommon manifestations, especially those with potentially life-threatening consequences such as Brugada syndrome. Performing a simple non-invasive test such as an ECG to patients with COVID-19 could be important for unmasking arrhythmias potentially related to the infection.

\section{Acknowledgments}

None to declare.

\section{Financial Disclosure}

None to declare.

\section{Conflict of Interest}

None to declare.

\section{Informed Consent}

Not applicable.

\section{Author Contributions}

MT Boncoraglio: writing original draft, literature review, and editing; J Esteves: literature review; F Pereira: review and editing; J Braga: literature review; C Veiga: literature review; D Oliveira: reviewed draft, stylistic and grammatical review; $\mathrm{P}$ Barbeito: supervision.

\section{Data Availability}

Any inquiries regarding supporting data availability of this study should be directed to the corresponding author.

\section{References}

1. Brugada J, Campuzano O, Arbelo E, Sarquella-Brugada G, Brugada R. Present status of Brugada syndrome: JACC State-of-the-Art review. J Am Coll Cardiol. 2018;72(9):1046-1059.

2. Bayes de Luna A, Brugada J, Baranchuk A, Borggrefe M, Breithardt G, Goldwasser D, Lambiase P, et al. Current electrocardiographic criteria for diagnosis of Brugada pattern: a consensus report. J Electrocardiol. 2012;45(5):433-442.

3. Baranchuk A, Nguyen T, Ryu MH, Femenia F, Zareba W, Wilde AA, Shimizu W, et al. Brugada phenocopy: new terminology and proposed classification. Ann Noninvasive Electrocardiol. 2012;17(4):299-314.

4. Bhatla A, Mayer MM, Adusumalli S, Hyman MC, Oh E, Tierney A, Moss J, et al. COVID-19 and cardiac arrhythmias. Heart Rhythm. 2020;17(9):1439-1444.

5. Driggin E, Madhavan MV, Bikdeli B, Chuich T, Laracy J, Biondi-Zoccai G, Brown TS, et al. Cardiovascular considerations for patients, health care workers, and health systems during the COVID-19 pandemic. J Am Coll Cardiol. 2020;75(18):2352-2371.

6. Stefanini GG, Montorfano M, Trabattoni D, Andreini D, Ferrante G, Ancona M, Metra M, et al. ST-Elevation Myocardial Infarction in Patients With COVID-19: Clinical and Angiographic Outcomes. Circulation. 2020;141(25):21132116.

7. Wang D, Hu B, Hu C, Zhu F, Liu X, Zhang J, Wang B, et al. Clinical characteristics of 138 hospitalized patients with 2019 novel coronavirus-infected pneumonia in Wuhan, China. JAMA. 2020;323(11):1061-1069.

8. Dherange P, Lang J, Qian P, Oberfeld B, Sauer WH, Koplan B, Tedrow U. Arrhythmias and COVID-19: a review. JACC Clin Electrophysiol. 2020;6(9):1193-1204.

9. Amin AS, Meregalli PG, Bardai A, Wilde AA, Tan HL. Fever increases the risk for cardiac arrest in the Brugada syndrome. Ann Intern Med. 2008;149(3):216-218.

10. van de Poll SWE, van der Werf C. Two patients with COVID-19 and a fever-induced Brugada-like electrocardiographic pattern. Neth Heart J. 2020;28(7-8):431-436.

11. Mahadevaiah G, Aleem A, Secaira A, Saba S, Shariff N. ST elevation in a patient with COVID-19 infectionassociated fever: a case of Brugada pattern. Cureus. 2020;12(6):e8722.

12. Pasqualetto MC, Corrado A, Secco E, Graceffa F, Rigo F. Transient type 1 Brugada pattern without ongoing fe- 
ver during COVID-19 pneumonia. Eur J Case Rep Intern Med. 2020;7(7):001808.

13. Lugenbiel P, Roth L, Seiz M, Zeier M, Katus HA, Merle
U, Schweizer PA. The arrhythmogenic face of COVID-19: Brugada ECG pattern during acute infection. Eur Heart J Case Rep. 2020;4(FI1):1-2. 